\title{
Prognostic significance of neutrophil-to-lymphocyte ratio in solid tumors: a note on methodological concerns
}

\author{
Masayuki Urabe*,1,2 (iD), Hiroharu Yamashita ${ }^{1} \&$ Yasuyuki Seto ${ }^{1}$ \\ ${ }^{1}$ Department of Gastrointestinal Surgery, Graduate School of Medicine, the University of Tokyo, Tokyo 113-8655, Japan \\ ${ }^{2}$ Department of Gastroenterological Surgery, Toranomon Hospital, Tokyo 105-8470, Japan \\ *Author for correspondence: Tel.: +813 3815 5411; Fax: +813 5800 9734; urabe-tky@umin.ac.jp
}
( Though NLR is generally accepted as a valuable prognostic tool in prognostication for cancer patients, issues regarding the methodology for stratifying pretreatment NLR have yet to be resolved."

First draft submitted: 2 September 2019; Accepted for publication: 13 September 2019; Published online: 10 October 2019

Keywords: inflammation $\bullet$ neutrophil-to-lymphocyte ratio $\bullet$ nonlinear $\bullet$ prognosis $\bullet$ solid tumor

A close link between inflammation and cancer was originally proposed more than a century ago by Rudolf Virchow, who in 1863 discovered leukocyte infiltration of malignant tissues and hypothesized that neoplasms originate from sites of chronic inflammation [1]. Dvorak further elaborated on this concept in 1986 by showing carcinogenic processes and inflammatory status to share common developmental pathways, suggesting that tumors appear to the host as wounds and described tumors as "wounds that do not heal" [2]. Ever since that description, the mechanisms underlying these hypothesized relationships have been actively investigated, and emerging lines of evidence have shown inflammatory and immunologic responses of the host to play essential roles in both the formation and the growth of tumors [3,4].

Accordingly, it is now widely accepted that systemic host responses to neoplasms have parallels with inflammatory conditions. Systemic immunologic responses have, though the exact mechanism remains uncertain, turned out to be potential markers for the progression of malignant diseases. The serum levels of some inflammatory parameters even reportedly correlate with the survival outcomes of patients. Among such inflammation-related indices, the neutrophil-to-lymphocyte ratio (NLR), the ratio of neutrophils to lymphocytes measured in the peripheral blood, is a well-known hematologic biomarker reflecting the systemic inflammation response.

\section{Association between pretreatment NLR \& clinical outcomes in patients with solid tumors}

The NLR was initially advocated as an easily measurable and available index of stress intensity and/or systemic inflammatory responses in critically ill patients receiving intensive care [5]. Walsh and colleagues underscored the good predictive ability of preoperative NLR for stratifying long-term outcomes of patients with colorectal cancer [6]. Thereafter, several research groups demonstrated the prognostic significance of pretherapeutic NLR for patients with various solid tumors [7]. The NLR elevation prior to treatment is reportedly associated with poorer outcomes for patients with esophagogastric cancer [8,9], colorectal cancer [6,10], hepatocellular carcinoma [11], pancreatic cancer [12], renal cell carcinoma [13], lung cancer [14], breast cancer [15] and many other malignancies [16]. Background factors explaining this clinical relevance of NLR in a broad range of cancers may include both increased neutrophil counts and decreased lymphocyte counts being involved in the processes of tumor cell proliferation. Cytokines generated by neutrophils, such as TNF- $\alpha$, IL-1, IL-6 and VEGF, have been described as creating a microenvironment facilitating angiogenesis and thereby promoting cancer cell growth and metastasis $[4,17]$. Moreover, tumor-associated neutrophils are also known to function as suppressors of the lymphocyte-mediated cytolytic activity, which plays a central role in antitumor immunity $[3,18]$. 
Therefore, higher NLR may well reflect an impaired immune response, with increased potential for tumor progression, and thus a poorer prognosis. Determining the NLR is easy and inexpensive, and the results are highly reproducible. These features of NLR have also attracted the attention of clinicians managing a variety of solid tumors.

\section{Challenging the conventional methodologies for prognostic stratification}

Though NLR is generally accepted as a valuable prognostic tool in prognostication for cancer patients, issues regarding the methodology for stratifying pretreatment NLR have yet to be resolved.

Templeton and colleagues reviewed the prognostic impact of NLR and evaluated its consistency in predicting the outcomes of patients with solid malignancies [16]. According to their large-scale review, most studies systematically included the post-treatment NLR in the final analysis as a categorical variable ( 95 of 100 studies), comparing clinical outcomes of two strata divided according to arbitrary cutoff values (mainly between three and five). Otherwise, in a small number of studies (4/100), NLR was assessed as a continuous variable in survival analysis using the Cox proportional hazards model. Whether NLR is assessed as a dichotomous or a continuous variable, these statistical methods are essentially based on the context that the correlation between mortality and pretreatment NLR can be estimated as monotonically linear or that NLR has a threshold effect on prognosis. However, previous studies did not seek to verify such tenuous assumptions.

A retrospective study performed by Koh and coworkers provided very interesting results [15]. They divided Asian women with breast cancer $(n=1435)$ into five groups based on quintiles of pretreatment NLR and compared long-term outcomes among these cohorts. Their Kaplan-Meier estimations demonstrated patients in the second quintile to have better long-term outcomes than those in the first quintile (5-year relative survival, 79.4 vs 76.4). This observation ran counter to the assumption that the prognostic correlation shows monophasic linearity.

Shimada et al., evaluating the utility of preoperative NLR for identifying patients with a poor prognosis after undergoing gastric cancer surgery $(n=1028)$, also raised the possibility of overall survival showing a nonlinear pattern [9]. Patients, whose NLR values were between 1.0 and 3.0, had more favorable outcomes than those with NLR values below 1.0 on Kaplan-Meier analysis. Although not statistically significant, this result also contradicted the 'monophasically linear' assumption.

In an effort to elucidate whether or not pretreatment NLR actually correlates with patient outcomes in a monophasic linear fashion, we performed a retrospective analysis of 1335 patients with gastric cancer, designed to determine the relationship between long-term outcomes and preoperative NLR [19]. The second quartile group showed significantly longer relapse-free survival rather than the first quartile group when multivariate Cox regression analysis was applied to NLR in combination with other influential prognostic factors (hazard ratio: 0.69; 95\% CI: $0.48-0.99 ; \mathrm{p}=0.048)$. This J-shaped relationship between the hazard ratios of relapse-free survival among the quartiles was supported by contrast testing (results similar to those were obtained in the analysis using the quintiles of NLR). This was the first study, to our knowledge, showing the association between the preoperative NLR values and long-term outcomes of gastric cancer patients to be nonlinear to a statistically significant degree.

\section{Next step: how should we employ NLR for predicting the outcomes of cancer patients?}

The aforementioned studies suggested a J-shaped relationship between survival time and pretreatment NLR in breast and gastric cancer patients. The results appeared to indicate an unfavorable prognosis for not only the highest NLR group but also the lowest NLR group. If true, treating NLR as a dichotomous variable would create a risk of ignoring prognostic heterogeneity. Employing 'continuous' NLR in survival analysis involves the same risk and should, therefore, perhaps not be recommended. In fact, research on patients with advanced bladder cancer, adopting clinical data from a major randomized Phase III trial (SWOG 8710), failed to demonstrate pretreatment 'continuous' NLR to be a reliable parameter predicting overall survival [20].

It is uncertain whether this suggestion can be universally applied to a broad range of solid malignancies, because the explicit rationale for the nonlinear prognostic pattern has yet to be established. Influences of patient background and life-style factors affecting neutrophil counts, for example, liver cirrhosis, drug usage and/or obesity, have been proposed but supporting evidence is lacking [19]. We can assert only that conventional methodologies should not be applied merely out of habit. As clinicians, caring for cancer patients, we need to rethink the most appropriate methods of using NLR as a prognostic indicator. 


\section{Conclusion}

The utility of NLR for predicting the outcomes of patients with malignancies has been extensively studied and several previous publications confirmed the prognostic value of NLR. However, in patients with some types of cancer, the association between pretreatment NLR and long-term outcome was suggested to be nonlinear. This finding raises the possibility that the conventional methodologies, such as applying NLR as a dichotomous or as a continuous variable, should be questioned. The nonlinearity of NLR observed herein may prompt careful reconsideration of how to best use this parameter in caring for our patients.

\section{Financial \& competing interests disclosure}

The authors have no relevant affiliations or financial involvement with any organization or entity with a financial interest in or financial conflict with the subject matter or materials discussed in the manuscript. This includes employment, consultancies, honoraria, stock ownership or options, expert testimony, grants or patents received or pending, or royalties.

No writing assistance was utilized in the production of this manuscript.

\section{Ethical conduct of research}

This study was approved by the ethics committee of the faculty of medicine, the University of Tokyo. All procedures performed in studies involving human participants were in accordance with the ethical standards of the institutional and/or national research committee and with the 1964 Declaration of Helsinki and its later amendments or comparable ethical standards.

\section{References}

1. Balkwill F, Mantovani A. Inflammation and cancer: back to Virchow? Lancet 357(9255), 539-545 (2001).

2. Dvorak HF. Tumors: wounds that do not heal. Similarities between tumor stroma generation and wound healing. $N$. Engl. J. Med. 315(26), 1650-1659 (1986).

3. Coussens LM, Werb Z. Inflammation and cancer. Nature 420(6917), 860-867 (2002).

4. Grivennikov SI, Greten FR, Karin M. Immunity, inflammation, and cancer. Cell 140(6), 883-899 (2010).

5. Zahorec R. Ratio of neutrophil to lymphocyte counts-rapid and simple parameter of systemic inflammation and stress in critically ill. Bratisl. Lek. Listy 102(1), 5-14 (2001).

6. Walsh SR, Cook EJ, Goulder F, Justin TA, Keeling NJ. Neutrophil-lymphocyte ratio as a prognostic factor in colorectal cancer. J. Surg. Oncol. 91(3), 181-184 (2005).

7. Zahorec R. Neutrophil-to-lymphocyte ratio. Sixteen-year-long history since publication of our article in Bratislava Medical Journal. Bratisl. Lek. Listy 118(6), 321-323 (2017).

8. Sato H, Tsubosa Y, Kawano T. Correlation between the pretherapeutic neutrophil to lymphocyte ratio and the pathologic response to neoadjuvant chemotherapy in patients with advanced esophageal cancer. World J. Surg. 36(3), 617-622 (2012).

9. Shimada H, Takiguchi N, Kainuma $\mathrm{O}$ et al. High preoperative neutrophil-lymphocyte ratio predicts poor survival in patients with gastric cancer. Gastric Cancer 13(3), 170-176 (2010).

10. Malietzis G, Giacometti M, Askari A et al. A preoperative neutrophil to lymphocyte ratio of 3 predicts disease-free survival after curative elective colorectal cancer surgery. Ann. Surg. 260(2), 287-292 (2014).

11. Mano Y, Shirabe K, Yamashita Y et al. Preoperative neutrophil-to-lymphocyte ratio is a predictor of survival after hepatectomy for hepatocellular carcinoma: a retrospective analysis. Ann. Surg. 258(2), 301-305 (2013).

12. Stotz M, Gerger A, Eisner F et al. Increased neutrophil-lymphocyte ratio is a poor prognostic factor in patients with primary operable and inoperable pancreatic cancer. Br. J. Cancer 109(2), 416-421 (2013).

13. Ohno Y, Nakashima J, Ohori M, Hatano T, Tachibana M. Pretreatment neutrophil-to-lymphocyte ratio as an independent predictor of recurrence in patients with nonmetastatic renal cell carcinoma. J. Urol. 184(3), 873-878 (2010).

14. Cannon NA, Meyer J, Iyengar P et al. Neutrophil-lymphocyte and platelet-lymphocyte ratios as prognostic factors after stereotactic radiation therapy for early-stage non-small-cell lung cancer. J. Thorac. Oncol. 10(2), 280-285 (2015).

15. Koh CH, Bhoo-Pathy N, Ng KL et al. Utility of pre-treatment neutrophil-lymphocyte ratio and platelet-lymphocyte ratio as prognostic factors in breast cancer. Br. J. Cancer 113(1), 150-158 (2015).

16. Templeton AJ, McNamara MG, Seruga B et al. Prognostic role of neutrophil-to-lymphocyte ratio in solid tumors: a systematic review and meta-analysis. J. Natl Cancer Inst. 106(6), dju124 (2014).

17. Kusumanto YH, Dam WA, Hospers GA, Meijer C, Mulder NH. Platelets and granulocytes, in particular the neutrophils, form important compartments for circulating vascular endothelial growth factor. Angiogenesis 6(4), 283-287 (2003).

18. Petrie HT, Klassen LW, Kay HD. Inhibition of human cytotoxic T lymphocyte activity in vitro by autologous peripheral blood granulocytes. J. Immunol. 134(1), 230-234 (1985). 
19. Urabe M, Yamashita H, Uemura Y et al. Non-linear association between long-term outcome and preoperative neutrophil-to-lymphocyte ratio in patients undergoing curative resection for gastric cancer: a retrospective analysis of 1335 cases in a tetrachotomous manner. Jpn J. Clin. Oncol. 48(4), 343-349 (2018).

20. Ojerholm E, Smith A, Hwang WT et al. Neutrophil-to-lymphocyte ratio as a bladder cancer biomarker: assessing prognostic and predictive value in SWOG 8710. Cancer 123(5), 794-801 (2017). 